\title{
Ajuste de Parámetros Cinéticos y Cálculo de sus Desviaciones usando Matlab ${ }^{\circledR}$
}

\author{
Santiago Zuluaga (1), Harold N. Ibarra(1), Izabela Dobrosz-Gómez ${ }^{(2)}$ y Miguel-Ángel Gómez ${ }^{(1)}$ \\ PRISMA: Grupo de Investigación en Procesos Reactivos Intensificados con Separación y Materiales \\ Avanzados. Universidad Nacional de Colombia, Sede Manizales. Campus La Nubia, Apartado Aéreo 127, \\ Manizales, Caldas, Colombia. (1) Departamento de Ingeniería Química, Facultad de Ingeniería y \\ Arquitectura; (2) Departamento de Física y Química, Facultad de Ciencias Exactas y Naturales. (e-mail: \\ sazuluagabo@unal.edu.co, hnibarrat@unal.edu.co, idobrosz-gomez@unal.edu.co,magomez@unal.edu.co)
}

Recibido Feb. 13, 2018; Aceptado Abr. 5, 2018; Versión final Jun. 8, 2018, Publicado Dic. 2018

\begin{abstract}
Resumen
El objetivo principal de este trabajo es enseñar la aplicación del método de mínimos cuadrados disponible en el software Matlab $^{\circledR}$ con sus comandos nlinfit y Isqcurvefit para determinar parámetros cinéticos, sus incertidumbres y las bandas asociadas a diferentes intervalos de confianza (95\% y 99\%). Para la ilustración de la metodología se emplearon datos cinéticos de tres procesos avanzados de oxidación, obtenidos experimentalmente, los cuales involucran: una única ecuación de velocidad de reacción en términos explícitos, una ecuación de velocidad de reacción en términos implícitos y un sistema de ecuaciones de velocidad de reacción en términos implícitos. Para cada uno se obtuvo los ajustes respectivos con diferencias significativas entre los parámetros y las desviaciones asociadas a los sistemas de pocos datos experimentales. En consecuencia, se ha demostrado que el tipo de modelo propuesto requiere de más valores experimentales para un buen ajuste u otro modelo que cumpla con los criterios lógicos y fenomenológicos esperados.
\end{abstract}

Palabras clave: mínimos cuadrados; regresión no lineal; bondad de ajuste; procesos avanzados de oxidación

\section{Fitting of Kinetic Parameters and their Deviations using Matlab ${ }^{\circledR}$}

\begin{abstract}
The main objective of this work is to teach the application of the least squares method available in the Matlab ${ }^{\circledR}$ software with its nlinfit and Isqcurvefit commands to determine kinetic parameters, their uncertainties and the bands associated with different confidence intervals (95\% and 99\%). To illustrate the methodology, kinetic experimental data obtained using three advanced oxidation processes, were fitted. Different approaches were used: a single equation of reaction velocity in explicit terms, an equation of reaction velocity in implicit terms and a system of reaction rate equations in implicit terms. For each one, the respective fittings were performed with significant differences between the parameters and the deviations associated with the systems with few experimental data. Consequently, it was shown that the type of proposed model required more experimental values for a good fit or another model that meets the expected logical and phenomenological criteria.
\end{abstract}

Keywords: least squares; non-linear regression; goodness of fit; advanced oxidation processes 


\section{INTRODUCCIÓN}

Uno de los principales temas de discusión a nivel mundial en el campo ambiental tiene relación con el crecimiento de la contaminación de las fuentes de agua por compuestos orgánicos o inorgánicos provenientes de actividades mineras, industriales y agrícolas (Moreira et al., 2017). Esta discusión ha fomentado el desarrollo y la difusión de nuevas tecnologías para el tratamiento de las aguas residuales cuando los tratamientos convencionales no son lo suficientemente eficientes. Tecnologías como los Procesos Avanzados de Oxidación (PAOs) ofrecen una interesante alternativa de tratamiento a esos compuestos recalcitrantes debido a que, dentro de su mecanismo de acción, los convierte en compuestos menos complejos y, en un escenario ideal, los conduce hacia la mineralización completa (Ribeiro et al., 2015). El estudio de la cinética química de estos procesos es un aspecto fundamental ya que permite conocer la relación que existe entre la reducción de contaminantes, el tiempo y las condiciones de reacción. Para representar dicha relación se han utilizado diversos modelos matemáticos que tienen en cuenta el cambio de concentración de la sustancia tóxica como tal (Li et al., 2005) o el cambio en algún parámetro fisicoquímico que determina su grado de polución (v.g., la Demanda Química de Oxígeno, DQO, la Demanda Biológica de Oxígeno, DBO, o la concentración de Carbón Orgánico Total, COT) en el transcurso del proceso (Gilpavas et al., 2016).

Un análisis cinético profundo de estos procesos requiere el uso de herramientas numéricas y computacionales poderosas (v.g., MatLab ${ }^{\circledR}$ ) que permitan determinar el comportamiento del proceso en su totalidad con el cálculo de parámetros cinéticos. Desde el punto de vista matemático, se define la forma de la ecuación que se pasará al proceso de iteración entre la/s variable/s dependiente/s e independiente/s hasta alcanzar una correspondencia adecuada utilizando el método probablemente más conocido y aplicado en la ingeniería química de mínimos cuadrados (Martínez et al., 2013). Finalmente, si ésta correspondencia no se cumple, definida a partir del análisis de la desviación de los parámetros y la amplitud de las bandas de confianza del ajuste, es necesario plantear otro modelo que permita describir el comportamiento observado o aumentar la cantidad de datos experimentales, si es posible, y correr el modelo inicial nuevamente.

En consideración de lo anterior, en este trabajo se presenta una metodología práctica, para su estudio y enseñanza en el aula, para realizar ajustes de datos cinéticos junto con la forma de determinar las incertidumbres tanto para los parámetros cinéticos como para el modelo matemático bajo estudio. Se presentan tres casos de estudio, con datos experimentales de PAOs para el tratamiento de aguas residuales, que permiten analizar igual número de situaciones de diferente grado de complejidad: i) una única ecuación de velocidad de reacción en términos explícitos, ii) una ecuación de velocidad de reacción en términos implícitos y iii) un sistema de ecuaciones en términos implícitos. Se muestra explícitamente como usar varias funciones predefinidas en MatLab ${ }^{\circledR}$ que permiten ajustar los parámetros de los modelos matemáticos y determinar la desviación de los parámetros cinéticos junto con sus bandas de confianza.

\section{MODELOS MATEMÁTICOS}

El algoritmo general para la determinación de los valores de parámetros, sus desviaciones y bandas de confiabilidad se esquematiza en la Fig. 1. Como variables de entrada se tienen las mediciones experimentales, el tipo de ajuste y las estimaciones iniciales de los parámetros a determinar. Con la selección del modelo se procede a escoger la función en MatLab ${ }^{\circledR}$ que permita la solución de los coeficientes y que suministre los residuales junto con la matriz Jacobiana para calcular las desviaciones y bandas de confianza asociadas al modelo.

Con respecto a la función de iteración, existen varias posibilidades. Su elección depende de los datos, modelo disponible y del funcionamiento de cada uno de ellos. En éste documento se muestra explícitamente el uso de dos comandos: nlinfit y Isqcurvefit. El primero de ellos es bastante útil para el caso de ajuste de una ecuación no lineal, mientras que el segundo ofrece varias ventajas cuando se tienen sistemas de ecuaciones no lineales. Los comandos mencionados buscan la minimización de la diferencia entre los parámetros medidos y los calculados a partir del modelo matemático propuesto, como lo ilustra la ecuación (1), siendo n el número de ecuaciones y $\mathrm{m}$ el número de variables independientes cuantificadas como $\mathrm{y}_{\mathrm{i}, \mathrm{j}}$ (con la variable $\mathrm{i}$ dependiente medida en la ecuación j y y ŷi, como la variable i dependiente estimada en la ecuación j). Por otra parte, los intervalos en los cuales se encuentran los parámetros ajustados, con una confianza determinada, son calculados con el software MatLab ${ }^{\circledR}$ usando comando nlparci, al cual se le debe suministrar, como condiciones de entrada, los parámetros ajustados, los residuales del sistema y la matriz Jacobiana asociada, extraídos directamente de las funciones iteradoras mencionadas. Éste comando funciona al aplicar la ecuación (2), donde bi es el parámetro hallado, $Z_{\alpha / 2}$ valor de la distribución normal a la confianza del sistema, $\sigma^{2}$ es la varianza y $\mathrm{J}$ es la matriz Jacobiana (Myers et al., 2010). 


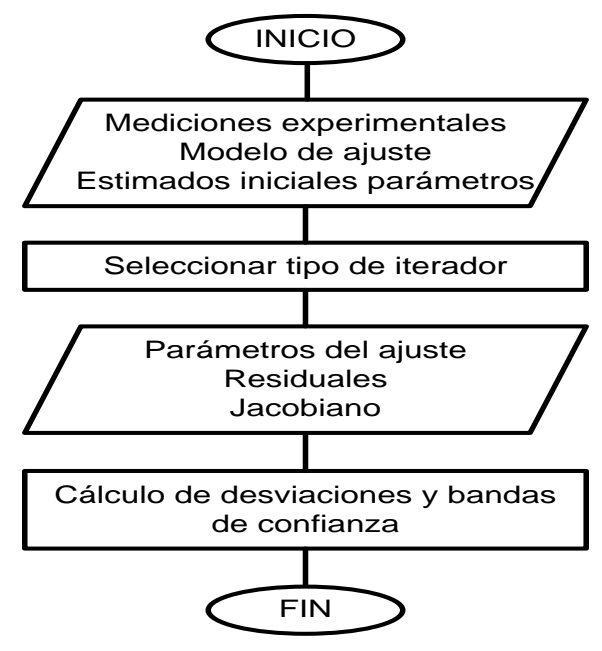

Fig. 1: Algoritmo de cálculo simplificado para el ajuste de modelos cinéticos

$$
\begin{aligned}
& \sum_{i=1}^{n} \sum_{j=1}^{m}\left(y_{i, j}-\hat{y}_{i, j}\right)^{2}=0 \\
& b_{i}-Z_{\alpha / 2} \sqrt{\sigma^{2}\left(J^{\prime} J\right)^{-1}} \leq \beta_{i} \leq b_{i}+Z_{\alpha / 2} \sqrt{\sigma^{2}\left(J^{\prime} J\right)^{-1}}
\end{aligned}
$$

Las bandas de confianza para una ecuación no lineal son fácilmente determinadas en MatLab ${ }^{\circledR}$ al aplicar el comando nlpredci, el cual necesita como condiciones de entrada la función que se ajustó, un vector $n$ de variables independientes definidas por el usuario para el intervalo de cálculo, los parámetros estimados y la matriz Jacobiana. Luego se extraen los valores predichos y el intervalo de cambio sobre el cual fueron calculados. Así, al evaluar la diferencia entre ellos, es posible determinar la banda inferior del sistema y al aplicar la adición se encontrará el comportamiento superior. Éste comando también funciona para sistemas de ecuaciones, pero es necesario reacomodar el sistema hacia las condiciones particulares del comando. Por ésta razón y en términos prácticos, luego del cálculo de los parámetros del proceso, resulta más sencillo aplicar la ecuación (3), puesto que requiere además de los parámetros contemplados para evaluar la ecuación (2), evaluar la función en los puntos medidos $\hat{y}\left(x_{0}\right)$ e ir ejecutando la matriz Jacobiana por filas $d_{o}($ Myers et al., 2010).

$$
\hat{y}\left(x_{o}\right)-Z_{\alpha / 2} \sqrt{\sigma^{2}\left(1+d_{o}^{\prime}\left(j^{\prime} J\right)^{-1} d_{o}\right)} \leq y_{o} \leq \hat{y}\left(x_{o}\right)+z_{\alpha / 2} \sqrt{\sigma^{2}\left(1+d_{o}^{\prime}\left(j^{\prime} J\right)^{-1} d_{o}\right)}
$$

Finalmente, entre mayor sea la cantidad de datos experimentales que se tengan, mejor será la estimación del comportamiento de las bandas; de otro modo, sería necesario la implementación de la matriz Jacobiana con el sistema de ecuaciones no lineales y su evaluación para el intervalo dependiente en que se quiera analizar del ajuste.

\section{CASOS DE ESTUDIO}

Para demostrar el uso de la metodología propuesta anteriormente, se pueden analizar en el aula de clases, con participación activa de los alumnos, tres casos de estudio relacionados con el tratamiento de aguas residuales (de la industria del café soluble y de la extracción del oro).

\section{Caso 1: Ajuste de datos a una única ecuación de velocidad de reacción en términos explícitos}

La industria del café soluble usa altas cantidades de agua durante las diversas etapas del proceso de producción. Consecuentemente, los efluentes generados se encuentran en alta proporción (entre 40 a $45 \mathrm{~L}$ por kilogramo de café tratado (Oller et al., 2011)) llevando consigo un alto poder contaminante como resultado de su alta carga orgánica, $\mathrm{pH}$ y color. En la búsqueda de tratamientos ideales de estas aguas residuales, Ibarra-Taquez et al. (2017) encontraron que la electro-coagulación (EC) y un PAO como la electro-oxidación (EO) son alternativas excelentes y efectivas para obtener altas remociones en DQO y COT. Para determinar el grado de descontaminación de un agua residual de la industria de café soluble, Ibarra-Taquez et al. (2017) cuantificaron la variación del COT en función del tiempo (t) (Tabla 1). 
Tabla 1. Datos experimentales del proceso secuencial EC - EO para aguas residuales de la industria del café soluble (Adaptada de lbarra-Taquez et al., 2017)

\begin{tabular}{|c|c|c|c|c|c|c|c|c|c|c|c|c|c|}
\hline Proceso & \multicolumn{4}{|c|}{ EC } & \multicolumn{10}{c|}{ EO } \\
\hline Tiempo $(\mathrm{min})$ & 0 & 15 & 30 & 45 & 60 & 75 & 90 & 105 & 120 & 135 & 150 & 165 & 180 \\
\hline COT $(\mathrm{mg} / \mathrm{L})$ & 1 & 0.793 & 0.702 & 0.66 & 0.6 & 0.542 & 0.506 & 0.497 & 0.475 & 0.432 & 0.41 & 0.382 & 0.365 \\
\hline
\end{tabular}

El proceso llevado a cabo fue realizado en un reactor por lotes, por lo tanto, la disminución del COT en función del tiempo se describe según la ecuación (4).

$$
\frac{\mathrm{dC}_{\mathrm{COT}}}{\mathrm{dt}}=-\mathrm{k} \mathrm{C}_{\mathrm{COT}^{n}}
$$

Separando variables, integrando y reemplazando las condiciones iniciales es posible obtener su solución analítica (ecuación (5)).

$$
\mathrm{C}_{\mathrm{COT}}=\left[-\mathrm{k}\left(\mathrm{t}-\mathrm{t}_{\mathrm{o}}\right)(1-\mathrm{n})+\mathrm{C}_{\mathrm{COT}_{\mathrm{o}}}{ }^{1-\mathrm{n}}\right]^{\frac{1}{1-\mathrm{n}}}
$$

donde C CoT la concentración de COT $(\mathrm{mg} / \mathrm{L}), \mathrm{k}$ es la constante de velocidad específica $\left(\mathrm{min}^{-1} .(\mathrm{mg} / \mathrm{L})^{1-\mathrm{n}}\right), \mathrm{t}$ tiempo (min), to tiempo inicial (min) y Cсотo la concentración de COT inicial ( $\mathrm{mg} / \mathrm{L}$ ). Para la determinación de los parámetros $\mathrm{k}$ y $\mathrm{n}$ del sistema, se aplicó el algoritmo de la Fig. 2, donde se usó como función de ajuste principal el nlinfit, que junto con el nlparci y nlpredci, permitieron el cálculo de las desviaciones de los parámetros y las bandas de confianza de todas las variables del proceso.

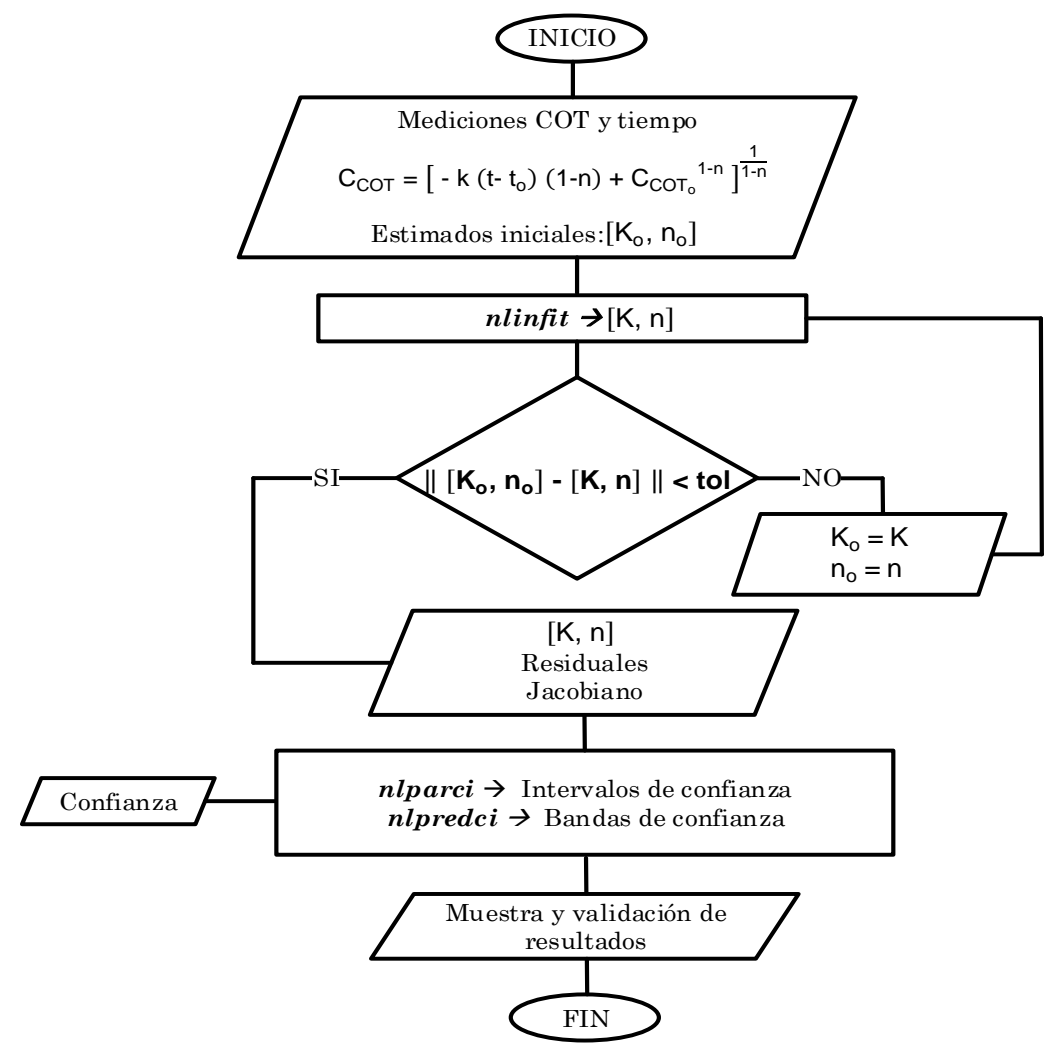

Fig. 2: Algoritmo de cálculo implementado para una ecuación no lineal

En la Fig. 3 se puede observar el comportamiento de los datos experimentales en función del tiempo, los resultados del modelo (línea sólida) y los parámetros cinéticos con su desviación asociada. Se incluyen también las bandas de confianza del ajuste respectivo para un intervalo de valores independientes definido de 0 a 60 minutos y de 60 a 180 minutos con 200 divisiones cada uno. En los dos casos se observa un comportamiento acorde de los modelos con respecto a los datos experimentales con parámetros cinéticos aceptables para el primer caso y bandas de confianza reducidas para el segundo caso. Esto se puede explicar considerando que el modelo propuesto para la EC es aceptable, en términos de las desviaciones de los 
parámetros, dado que la amplitud de las bandas no supera el valor hallado pese a la cantidad reducida de datos experimentales tomados en el intervalo. Por otra parte, el modelo de EO presenta una desviación alta de los parámetros, indicando la necesidad de simplificar el orden del modelo (por ejemplo, de tipo potencial a lineal) y del ancho de bandas. En síntesis, el modelo para el proceso de EC puede ser más predictivo, según la desviación obtenida de los parámetros, y de mayor incertidumbre si es comparado con el proceso de EO.

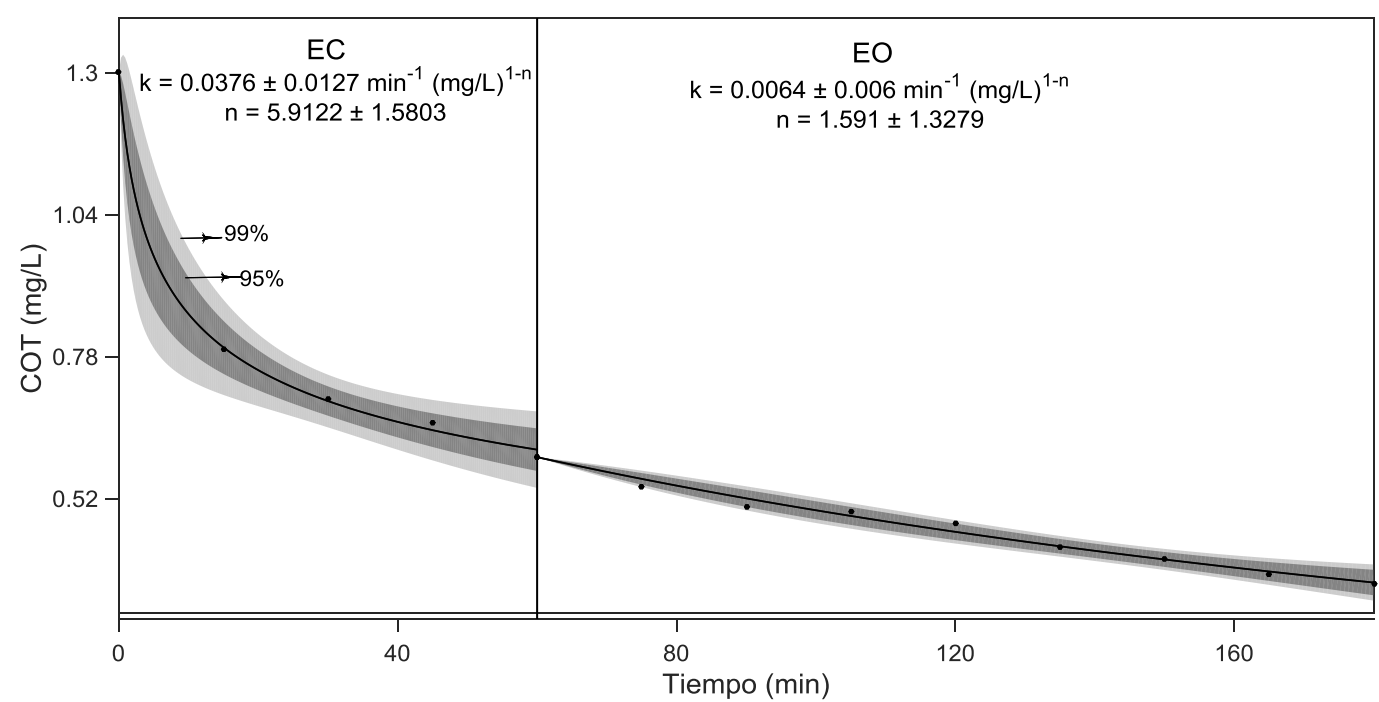

Fig. 3: Ajuste de datos experimentales con bandas de confiabilidad del 99 y $95 \%$ del sistema de EC - EO para tratamiento de aguas residuales provenientes de industria de café soluble

\section{Caso 2: Ajuste de datos a una ecuación de velocidad de reacción en términos implícitos}

El uso del cianuro para la extracción de metales preciosos es un método muy utilizado a nivel industrial. Sin embargo, se debe remover o mineralizar de los efluentes la cantidad remanente de cianuro ya que este puede representar riesgos ambientales para el ser humano y la vida animal. En los últimos años han aumentado los estudios encaminados a establecer posibles alternativas de solución. Entre ellas, Ramos (2016) definió una forma de remoción de cianuro a partir de la aplicación de otro PAO como fotocatálisis heterogénea. Este permite la mineralización del cianuro por su interacción con los radicales hidroxilo $\left(\mathrm{OH}^{*}\right)$ generados por el catalizador de $\mathrm{TiO}_{2}$ y radiacion UV, en presencia de peróxido de hidrógeno. La reacción de fotocatálisis heterogénea para la remoción de cianuro se presenta en la ecuación (6). Se puede observar que a partir del cianuro $\left(\mathrm{CN}^{-}\right)$y radical hidroxilo $\left(\mathrm{OH}^{*}\right)$, se forman dos sustancias menos tóxicas como lo son el cianato (CNO-) y $\mathrm{HO}_{2}^{*}$. Ramos (2016) propuso modelar la reacción usando una expresión tipo Langmuir-Hinshelwood junto con el balance molar en un reactor por lotes (ecuación (7)).

$$
\begin{aligned}
& \mathrm{CN}^{-}+\mathrm{OH}^{*} \rightarrow \mathrm{CNO}^{-}+\mathrm{HO}_{2}{ }^{*} \\
& \frac{\mathrm{dC}_{\mathrm{CN}^{-}}}{\mathrm{dt}}=\frac{-\mathrm{kKC}_{\mathrm{CN}^{-}}}{1+\mathrm{KC} \mathrm{CN}_{\mathrm{CN}^{-}}}
\end{aligned}
$$

En éste caso de estudio, es necesaria la solución de la ecuación diferencial, usando una de las funciones ode (escogida dependiendo la complejidad) junto con el comando Isqcurvefit de MatLab ${ }^{\circledR}$. De ésta manera, es posible determinar las constantes $\mathrm{k}$ y $\mathrm{K}$ del modelo. El algoritmo de solución planteado para éste sistema se presenta en la Fig. 4. Notese que además del ingreso de los valores de las parejas de datos de concentración vs. tiempo (Tabla 2), es necesario crear una función externa que contenga la ecuación diferencial (ecuación 7). Su solución alimentará al iterador para proponer un nuevo vector de parámetros de solución que serán comparados con los propuestos inicialmente hasta su convergencia.

Tabla 2. Datos experimentales de cambios de concentracion de $\mathrm{CN}^{-}$vs. tiempo usando fotocatálisis heterogénea, como tratamiento de aguas residuales provenientes de la extracción de oro con cianuro (adaptados de Ramos (2016))

\begin{tabular}{|c|c|c|c|c|c|c|}
\hline Tiempo (min) & 0 & 25 & 50 & 75 & 100 & 125 \\
\hline Concentración de $\mathrm{CN}^{-}(\mathrm{mg} / \mathrm{L})$ & 102.73 & 51.38 & 35.57 & 23.71 & 13.83 & 5.93 \\
\hline
\end{tabular}


Seguido, es posible determinar los intervalos de confianza asociados y, aplicando la ecuación (3), las bandas de confianza del ajuste respectivo. La Fig. 5 presenta los resultados de aplicación del algoritmo (Fig.4) para resolver la ecuación (7). Los resultados del modelo obtenidos presentan una alta desviación de los parámetros y bandas de confianza amplias a lo largo del intervalo, lo que indica directamente que el modelo escogido por el autor no es adecuado para tener correspondencia con los datos experimentales. Marugan et al. (2006) sugirieron tener también en cuenta la influencia de la irradiación en el sistema dependiendo la geometría que tenga el reactor; dicha expresión puede mejorar la solución del sistema pero aumentaría la cantidad de parámetros a determinar y con 6 datos experimentales la incertidumbre de los parámetros permanecerá. Por ésta razón, y para tener un mejor comportamiento del modelo, se hace necesario incrementar la cantidad de datos experimentales, o reportar los valores con las réplicas establecidas y ejecutar de nuevo el algoritmo.

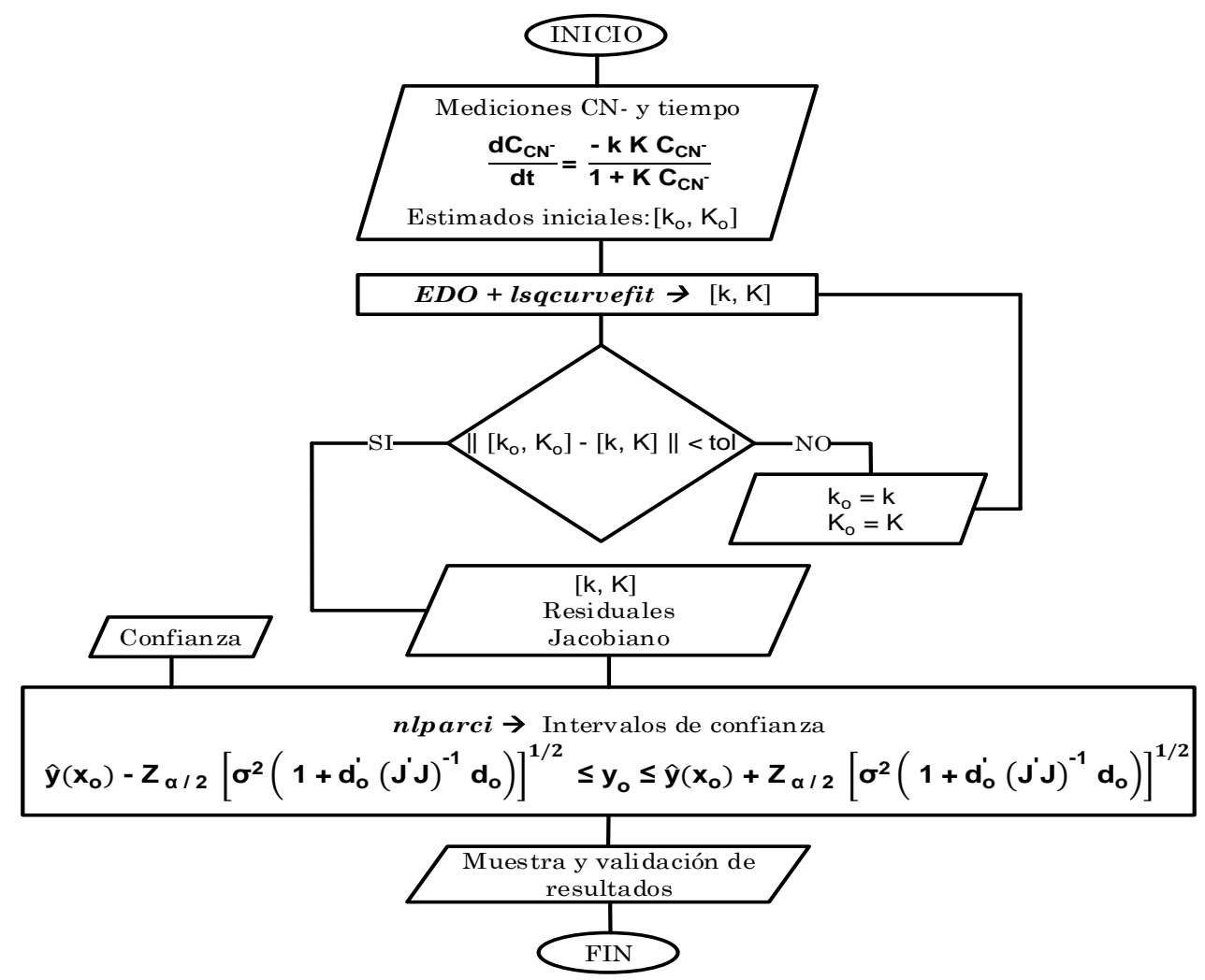

Fig. 4: Algoritmo de cálculo implementado para una ecuación diferencial

Es importante destacar que cuando se tiene una desviación de los parámetros alta, las bandas de confianza tienden a ser mas gruesas todo debido a que el cálculo de los valores predictivos va ligado directamente a la varianza del sistema como se observa en la ecuación (3).

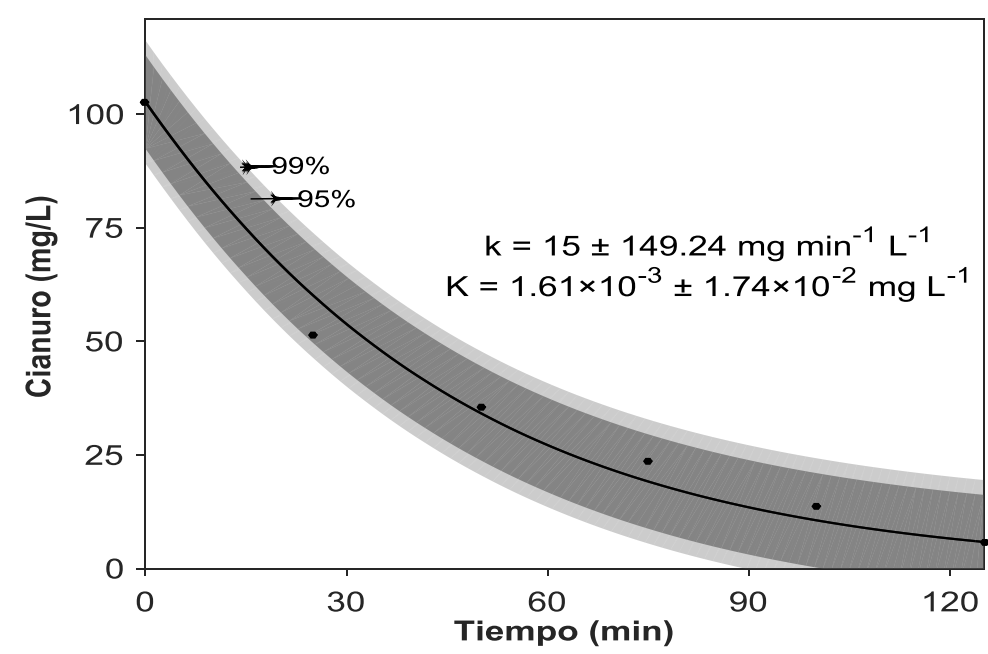

Fig. 5: Ajuste de datos experimentales con bandas de confiabilidad del 99 y $95 \%$ del sistema de fotocatálisis heterogénea para tratamiento de aguas residuales provenientes de la extracción de oro con cianuro 


\section{Caso 3: Ajuste de datos a un sistema de ecuaciones de velocidad de reacción en términos implícitos}

En línea con lo expuesto en el Caso 2, Gaviria-López (2016) evaluó la mineralización del cianuro de las aguas residuales aplicando oxidación electroquímica, proceso contemplado dentro de los tipos PAOs existentes. Tanto en la EO como en la fotocatálisis mencionada el actor principal de remoción es el radical hidroxilo, el cual por su estructura química es altamente oxidativo, sobre todo si es impulsado por una corriente eléctrica fomentada por un par de electrodos conectados a una fuente de poder. Gaviria-López (2016) realizó el seguimiento del sistema reactivo teniendo en cuenta tanto la producción de cianato como la desaparición de cianuro en función del tiempo de reacción. De esta forma, considerando cinéticas del tipo ley de potencias, fue posible obtener las ecuaciones (8) y (9) para un reactor por lotes.

$$
\begin{aligned}
& \frac{\mathrm{dC}_{\mathrm{CN}^{-}}}{\mathrm{dt}}=-\mathrm{k}_{\mathrm{CN}^{-}} \mathrm{C}_{\mathrm{CN}^{-}}{ }^{\alpha} \\
& \frac{\mathrm{dC}_{\mathrm{CNO}^{-}}}{\mathrm{dt}}=\mathrm{k}_{\mathrm{CN}^{-}} \mathrm{C}_{\mathrm{CN}^{-}}{ }^{\alpha}-\mathrm{k}_{\mathrm{CNO}^{-}} \mathrm{C}_{\mathrm{CNO}^{-}}{ }^{\beta}
\end{aligned}
$$

Con el sistema de ecuaciones diferenciales definido y los datos experimentales reportados en la Tabla 3 , se implementó el algoritmo presentado en la Fig. 6. En este caso, además de ingresar todos los datos experimentales, fue necesario generar una función externa que contenga el sistema de ecuaciones diferenciales y los valores de iteración iniciales para comenzar su ejecución. Si bien es necesario determinar para cada valor de tiempo las concentraciones correspondientes con los estimados propuestos, también se requiere de una comparación entre las concentraciones halladas y las medidas.

Tabla 3. Datos experimentales de cambios de concentracion de $\mathrm{CN}^{-}$y $\mathrm{CNO}^{-}$vs. tiempo en aguas residuales provenientes de extracción de oro con cianuro (adaptados de Gaviria-López, 2016)

\begin{tabular}{|c|c|c|c|c|c|}
\hline Tiempo (min) & 0 & 5 & 10 & 15 & 17 \\
\hline Concentración de $\mathrm{CN}^{-}(\mathrm{mg} / \mathrm{L})$ & 97.27 & 60.18 & 39 & 19.45 & 9.73 \\
\hline Concentración de CNO $(\mathrm{mg} / \mathrm{L})$ & 0 & 46.08 & 57.12 & 57.24 & 26.94 \\
\hline
\end{tabular}

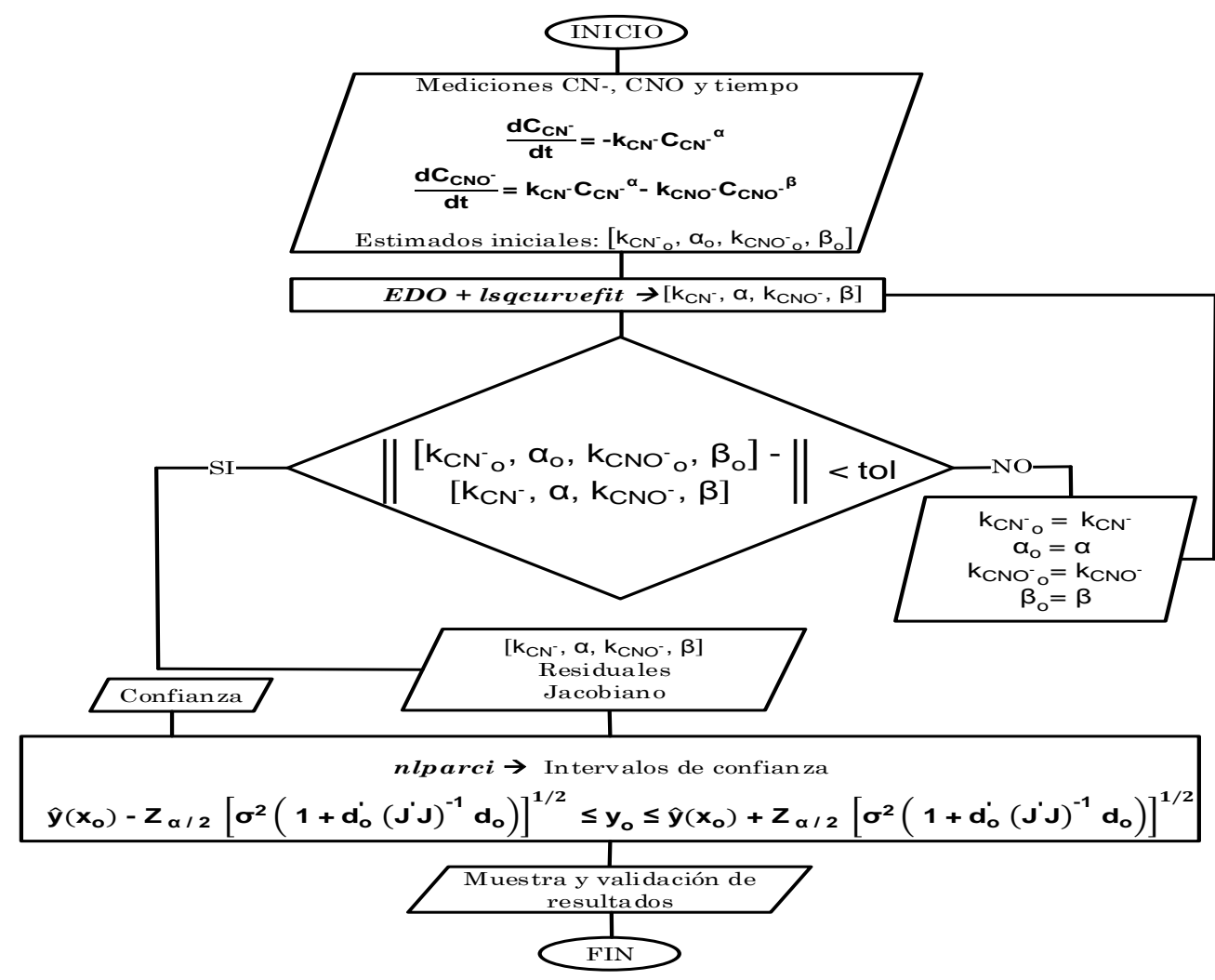

Fig. 6: Algoritmo de cálculo implementado para un sitema de ecuaciones diferenciales 
Así, se toma nuevamente importancia el uso del comando Isqcurvefit usado en el caso anterior, pues permite tener una convergencia adecuada. Con la iteración continua por parte del comando y el algoritmo es posible determinar los cuatro parámetros desconocidos para el sistema y así especificar la cinética de reacción del proceso de EO (Fig. 7). Se observa un comportamiento similar al reportado en el caso 2, dado que en cuatro de los tres parámetros cinéticos determinados la desviación de los mismos es superior al valor hallado, y sería necesario eliminar los parámetros ambiguos y resolver de nuevo el modelo, haciéndose el sistema de ecuaciones diferenciales inconsistente respecto a lo mostrado en la Tabla 3. Ésta situación se sustenta también en la amplitud de las bandas de confianza, las cuales se hacen gruesas debido a la cantidad reducida de datos experimentales aportados por el autor. En síntesis, el modelo presentado por el autor requiere de un análisis más riguroso como lo reportan Li \& Liu (2009) donde para la constante de velocidad de degradación del cianuro tienen en cuenta la concentración del electrolito y la densidad de corriente establecida.

\section{INFORME DESARROLLADO POR LOS ALUMNOS}

Para un aprendizaje conciso y efectivo sobre el ajuste de datos cinéticos el estudiante debe plantear los balances de materia para el tipo de reactor involucrado. Posteriormente, acoplando el tipo de ley de velocidad debe seleccionar el método de solución para la determinación de las constantes cinéticas. Finalmente, debe proceder a la programación respectiva, sugiriéndose aplicar el algoritmo general presentado en la Fig. 1, empezando por el ingreso de los datos experimentales, pasando por la generación de la función objetivo y, dependiendo la complejidad del modelo matemático involucrado, escoger el iterador adecuado. Al obtener los valores esperados se comprarán los parámetros con las desviaciones asociadas, si las desviaciones son menores que los parámetros se continúan los cálculos de las bandas de confianza, sino se propone identificar otro modelo y realizar el desarrollo mencionado anteriormente.

\section{CONCLUSIONES}

Con la aplicación del método de mínimos cuadrados con el software MatLab ${ }^{\circledR}$ se mostró como realizar las regresiones no lineales para los tres sistemas reactivos de tratamiento de aguas residuales industriales de diferente grado de complejidad. Se determinaron los parámetros de ajuste, intervalos y bandas de confianza. En el primer caso de estudio se observó una tendencia del modelo acorde al proceso de EC llevado a cabo, ya que cumple con los requerimientos establecidos para un buen modelo, con desviaciones acordes a los valores de los parámetros y tendencia definida sobre los valores experimentales. Por otra parte, los resultados de EO del primer proceso y los casos siguientes presentaron grandes desviaciones e incertidumbres tanto de sus parámetros como de sus modelos. Se mostró que este tipo de resultado requiere de una profundización y análisis alternativo de cinéticas que contemplen con más rigurosidad el fenómeno presentado y un aumento de datos experimentales para obtener una mejor correspondencia entre ellos.

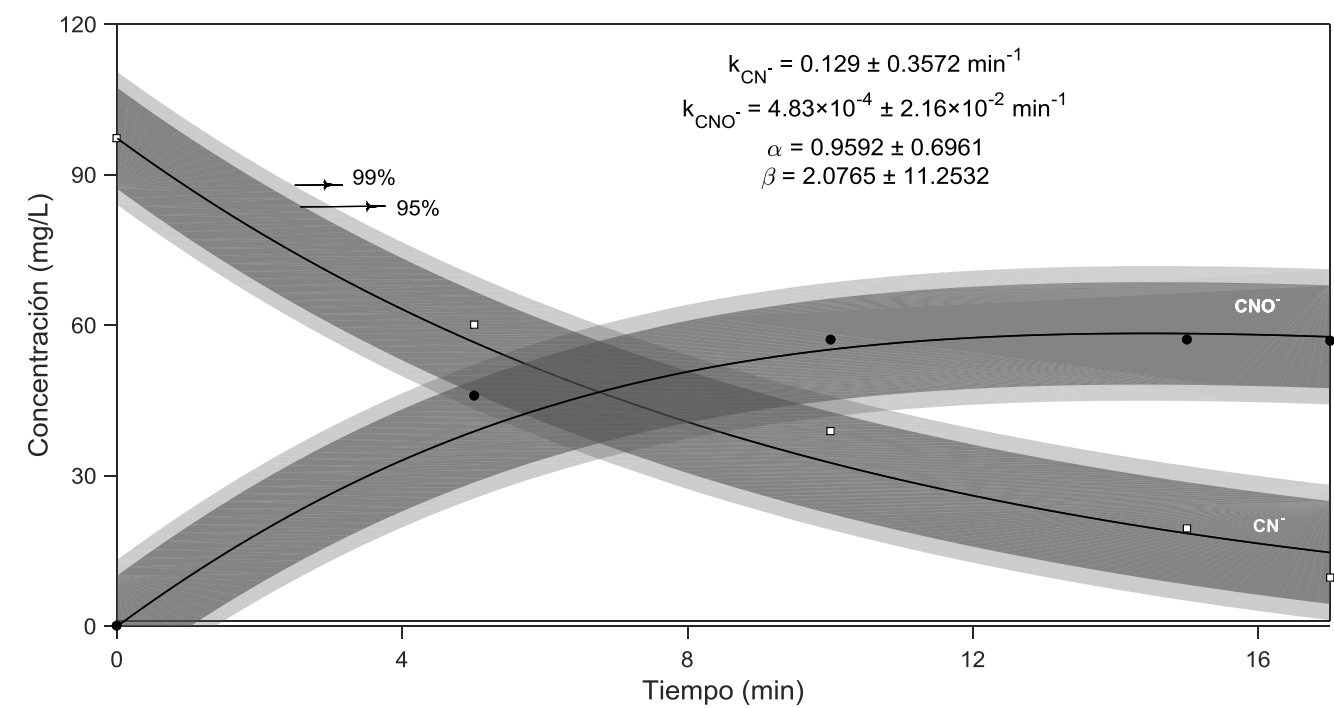

Fig. 7: Ajuste de datos experimentales con bandas de confiabilidad del 99 y $95 \%$ del sistema de EO para tratamiento de aguas residuales provenientes de la extracción de oro con cianuro

\section{AGRADECIMIENTOS}

Los autores agradecen al profesor Diógenes de Jesús Ramírez Ramírez por las fructíferas discusiones de conceptos estadísticos. Harold lbarra agradece a Colciencias por su apoyo económico (Convocatoria Nacional 567 para estudios de Doctorado en Colombia año 2012). 


\section{REFERENCIAS}

Gaviria-López, G. H., Tratamiento de Aguas Residuales del Proceso de Lixiviación de Oro con Cianuro a través de Oxidación Electroquímica, Tesis de Maestría en Ingeniería Química, Universidad Nacional de Colombia, Sede Manizales (2016)

Gilpavas, E., J. Medina y otros dos autores, Degradación de Colorante Amarillo 12 de Aguas Residuales Industriales utilizando Hierro Cero Valente, Peróxido de Hidrógeno y Radiación Ultravioleta, doi:10.4067/S0718-07642016000300004, Inf. Tecnol., 27, 23-34 (2016)

Ibarra-Taquez, H. N., E. GilPavas y otros tres autores, Integrated Electrocoagulation-Electrooxidation Process for the Treatment of Soluble Coffee Effluent: Optimization of COD Degradation and Operation Time Analysis, doi: 10.1016/j.jenvman.2017.05.095, J. Environ. Manag., 200, 530-538 (2017)

Li, L. e Y. Liu, Ammonia removal in electrochemical oxidation: Mechanism and pseudo-kinetics, doi: 10.1016/j.jhazmat.2008.04.047, J. Hazard. Mater, 162, 1010-1016 (2009)

Li, Y.C., L.G. Bachas y D. Bhattacharyya, Kinetics Studies of Trichlorophenol Destruction by Chelate-Based Fenton Reaction, doi:10.1089/ees.2005.22.756, Environ. Eng. Sci., 22, 756-771 (2005)

Martínez, J. J., H. Rojas y P. Reyes, Kinetic Study of the Hydrogenation of Citral on Ir Promoted Au/TiO2 Catalyst, doi: 10.4067/S0717-97072013000300001, J. Chil. Chem. Soc., 58(3), 1799-1804 (2013)

Marugan, J., R. Van-Grieken y otros dos autores, Optical and Physicochemical Properties of Silica-Supported TiO 2 Photocatalysts, doi: 10.1002/aic.10886, AIChE J., 52(8), 2832-2843 (2006)

Moreira, F., R. Boaventura y otros dos autores, Electrochemical Advanced Oxidation Processes: A Review on their Application to Synthetic and Real Wastewaters, doi: 10.1016/j.apcatb.2016.08.037, Appl. Catal. B. Environ., 202, $217-261$ (2017)

Myers, R., D. Montgomery y otros dos autores, Generalized Linear Models with Applications in Engineering and the Sciences, 2 ${ }^{\mathrm{a}}$ Ed., John Willey \& Sons, Estados Unidos de América (2010)

Oller, I., S. Malato y J.A. Sánchez-Pérez, Combination of Advanced Oxidation Processes and biological treatments for wastewater decontamination: a review, doi: 10.106/j.scitotenv.2010.08.061, Sci. Total Environ., 409(20), 4141-4166 (2011)

Ramos, B. D., Oxidación Fotocatalítica como Alternativa de Tratamiento de Aguas Residuales del Proceso de Extracción de Oro con Cianuro, Tesis de Maestría en Ingeniería Química, Universidad Nacional de Colombia, Sede Manizales (2016)

Ribeiro, A. R., O. C. Nunes y otros dos autores, An Overview on the Advanced Oxidation Processes Applied for the Treatment of Water Pollutants, doi:10.1016/j.envint.2014.10.027, Environ. Int., 75, 33-51 (2015) 
